\title{
Students' motivation, concentration and learning skills using Augmented Reality
}

\author{
Sampaio, Daniel and Almeida, Pedro
}

Digimedia - Universidade de Aveiro, Portugal

\begin{abstract}
The use of new technologies in the teaching and learning process, as the case of devices with the use of Augmented Reality, captures student and teacher's attention, creating the expectation that its use can provide the participants with new ways of interacting, new possibilities for collaboration and potentially an increase in motivation for learning. This research promoted the use of Augmented Reality using four prototypes created by the researcher/teacher during the teaching-learning process and had as main objectives: to evaluate the impact on motivation and learning competencies; identify, explore and evaluate different strategies for the integration of Augmented Reality devices and resources and define a set of guidelines for the integration of augmented reality systems. With the results of the questionnaires completed online by the students, it was concluded that they were greatly interested in the possibility of interacting this way with the programmatic contents, the use of the prototypes added a greater motivation to solve the tasks proposed by the teacher and, during the use of the prototypes, the students showed high levels of concentration for their achievement.
\end{abstract}

Keywords: Augmented Reality; Student Motivation/ Concentration; Learning strategies; Information and Communication Technologies; Educational Technologies. 


\section{Introduction}

The success index of the courses is many times affected by the student's (lack of) motivation and by difficulties in learning with the content. According to Brophy (2004) the concept of student motivation is used to explain the degree to which students devote their attention and effort in various activities that may or may not be the level desired by their teachers. The introduction of new technologies, in this context, can be an important asset to overcome these difficulties and create the conditions of change that may involve the student into more intensive learning experiences. It is important for an educational system to be upto-date with the technological advances and the changes it may bring to teaching innovation and as a complement to the learning process in the classroom. The technologies that are being gradually introduced in educational contexts enable teachers and students to diversify strategies that lead to knowledge building so it will be necessary to understand what advantages they can bring to the teaching-learning process. As Joseph (2012) points out, technology will continue to dominate many aspects of human existence and, if used to full potential, can guarantee an improvement in the teaching-learning process, leading to the need of understanding whether the impacts of the technological education are more positive in comparison with traditional forms of teaching. Thus, the exploitation of new technologies in the classroom is always a challenge for all participants in the teachinglearning process. If on the one hand the students possess a high digital literacy, due to being immersed in a society addicted to technology, on the other hand, the teachers are not always up to par in handling this technology; although there are, for both teachers and students, many advantages, as pointed out by Sobral and Menezes (2012), when they state that the use of technologies in the classroom increases the motivation of the students, their concentration is higher and, as a consequence, they learn more. Therefore, the student/teacher relationship becomes more in tune. Education is still unable to keep up with the constant changes that technology has had, as in many areas, in education, Information and Communication Technologies (ICT) has enormous potential of exploitation which can be an advantage for student learning and consequently in their educational success. For that, it will be necessary to explore the teaching-learning processes using these technologies. One of these technologies, Augmented Reality (AR), has captured the attention of students and teachers. As indicated by Nincarean et al (2013) AR is one of the emerging technologies that can have enormous potential and impact on learning. According to Shelton and Hedley (2002), the arrival of new technologies, such as AR systems, captures the attention also for pedagogical purposes. According to Kesim and Ozarslan (2012) the students when using AR technology interact with objects and information in three dimensions in a natural way. In the current context, where the use of AR devices has been intensifying in education, the learning process can be transformed. Although, the introduction of technologies in the classroom can create new ways of knowledge 
acquisition, it is necessary to evaluate if they have the same skills of a traditional manual or book. Di Serio, Ibáñez and Kloos (2013) in a usability study have shown that, although AR is not yet sufficiently used in education, student enthusiasm has reduced most of the difficulties, and this may be a determining factor for the student's growing interest in pedagogical content and the effective success of the introduction of this type of technology in education.

\section{Research Purpose}

\subsection{Objectives}

The project has three main goals:

- Evaluate if the introduction of Augmented Reality technologies in the learning process carries significant improvements in the motivation and competences acquisitions of students;

- Identify, explore and evaluate different strategies for the integration of Augmented Reality systems and its features in the teaching and learning process of an Information Communication Technologies (ICT) subject;

- Define a set of guidelines (best practices) for the integration of Augmented Reality systems in teaching and learning processes.

\subsection{Methodology}

This research project has a sample of sixty-two students from three 8th grade classes of the Agrupamento de Escolas D. Pedro IV, Vila do Conde. These students do not have prior experience with Augmented Reality systems and most of them not even know about this type of technology. This project follows an Action Research methodology since it includes several research cycles focused on the development and evaluation of different Augmented Reality based prototypes and related teaching strategies for its integration in the ICT curriculum. Each student used the 4 prototypes (Figure 1) while solving a worksheet and was invited to answer a brief questionnaire after each session. Following the Action Research methodology, the planning of the four prototypes took in consideration the conclusions regarding the use of the previous cycle. 

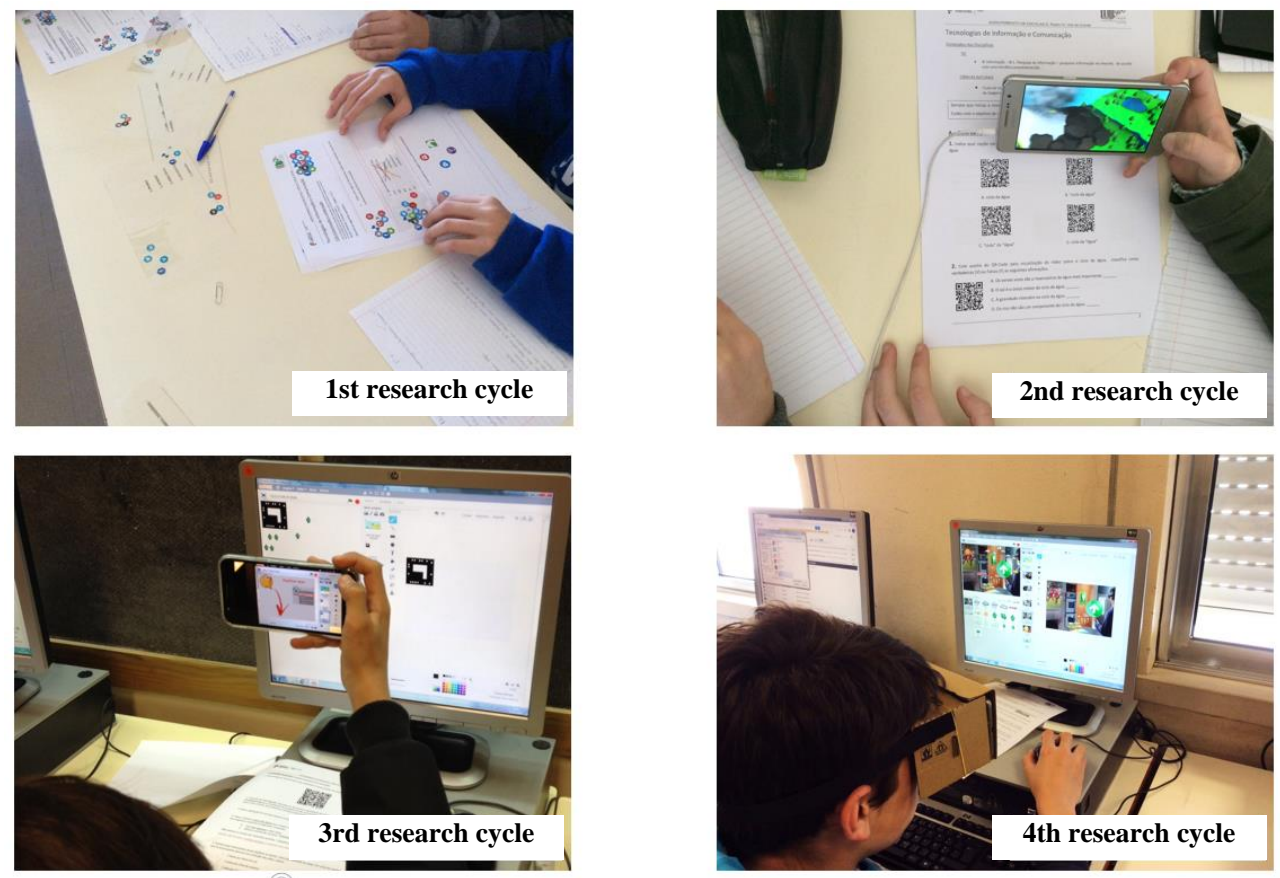

Figure 1. Research cycles

\subsubsection{First research cycle}

In the first research cycle the prototype was built with the objetive of creating a parallelism between transparencies (acetate) over printed assignments with the foundations of a device with an AR system that integrates a real object with a virtual in a real environment. The transparencies contained printed solutions and when overlaid on an assignment paper revealed the solution for the assignment. Despite the fact that this prototype was prepared with paper and acetates, the main goal was to prepare students to work with systems that expand reality, complementing it with tips and solutions, in a simplistic way, but in essence close to what can be obtained with an AR system.

\subsubsection{Second research cycle}

The second prototype was targeted to an assignment that integrated contents from ICT and Natural Sciences. It was focused on (information) search techniques and materials flow in ecosystems. This prototype was the first to use mobile devices. Students had to install an application to scan for QR-Codes (QRC). When they did it, it showed multimedia content linked to each question of the paper assignment. 


\subsubsection{Third research cycle}

The third cycle was based on the idea that the student will be pointing the mobile device to a real object and will be able to visualize the virtual elements related with the programmatic contents, i.e., use integrally the AR system in their devices. In this cycle it was possible to make a parallel between the way QRC and AR work, because in the second cycle students using QRC had access in the mobile device to a hyperlink with multimedia contents but in the third cycle the multimedia contents were displayed on the devices above the actual object that was scanned. When solving the tasks the contents remained related to ICT and Natural Sciences. The student, in order to solve the tasks, had to follow the provided steps to see on the screen of the mobile device the multimedia contents that helped with the task completion.

\subsubsection{Fourth research cycle}

It also took in consideration the idea that this prototype should make use of a complete and immersive AR system. It differed from the third prototype because it was developed so that the student could work "freely" with the keyboard and mouse without having to hold the mobile device to watch the contents in the AR system. Although with similar concepts of the third prototype, it introduced a Google Cardboard placed on the head using a tape. This way the student had an opened field of view, focused on the contents that appeared on the screen, and was able to have his hands free to work. It was intended with this prototype to make a simulation of the effective use of AR glasses, because it is an emergenttechnology and still has quite high acquisition values. However, using Google Cardboard if paired with a mobile device with an AR system installed, despite the lower quality, it integrates the principles of AR glasses.

\subsection{Results}

At the end of each research cycle the students completed an online questionnaire related with the use of the prototype. Of the several results obtained, the following are highlighted:

Graph 1 shows the high value of students' motivation using the prototypes. In particular, the values of the last two prototypes that use AR are very high, above 90\%. Thus, students' motivation increased with the introduction of the prototypes using AR (prototype 3 and 4) in relation to the two previous prototypes (prototype 1 and 2) that didn't have this technology. 


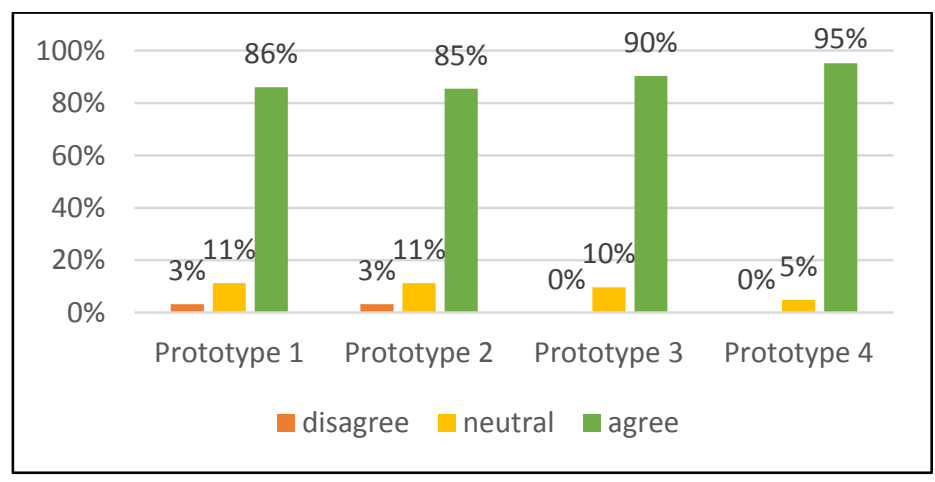

Graph 1. Were the students motivated using the prototype?

As can we see in Graph 2, concerning the comprehension of contents by students, they clearly agreed in their answers, with values between $73 \%$ (P4) and 81\% (P2/P3), which shows that the introduction of AR in the teaching-learning process can increase the success index of student learning.

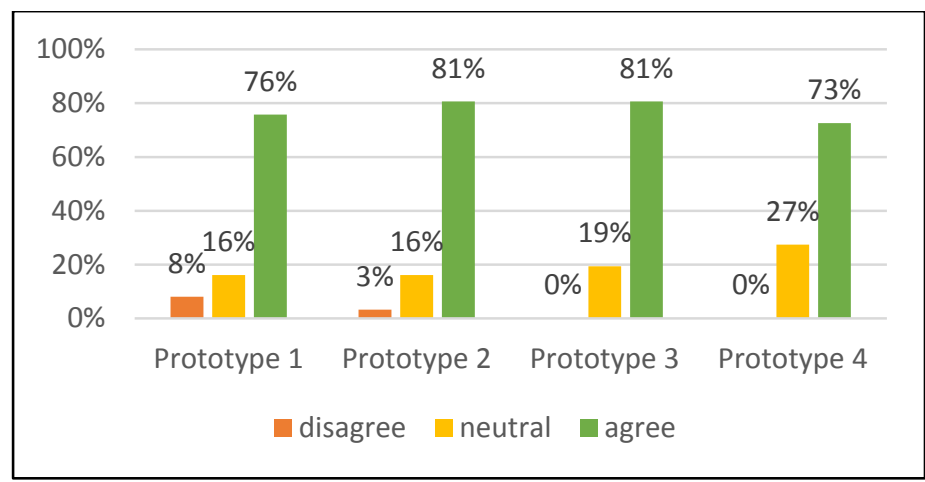

Graph 2. Did the students understand the content using the prototypes?

Graph 3 shows the student concentration perception while solving the exercises using the prototypes. We can verify a perception of high concentration levels. However, it is important to mention the decrease in student concentration from the first to the fourth prototype, but the concentration values are still high. This decrease can be related to the distraction that the use of a technological innovation can cause in the students and by the fact that Google Cardboard (prototype 4) occupied the field of vision of the students, creating a filter in the perception of the reality. However, it is important to note that this small decrease in student concentration is not correlated with student motivation. It is also important to highlight the slight decrease in student concentration in contrast to the increasing introduction of technology in the prototype. Based on this, we can conclude that 
adding more technology reduces student concentration, but their motivation increases significantly.

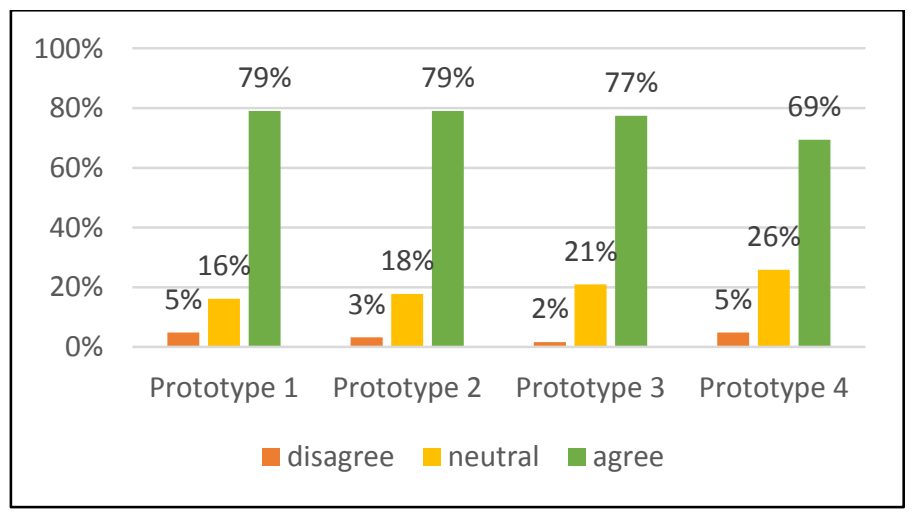

Graph 3. Were the students concentrated using the prototype?

Graph 4 expresses the students' opinion about the advantages of visualizing the contents with Augmented Reality Glasses (ARG) while working/studying. It is possible to conclude, from the presented values (73\%), that students clearly express their agreement with the use of ARG in an educational context.

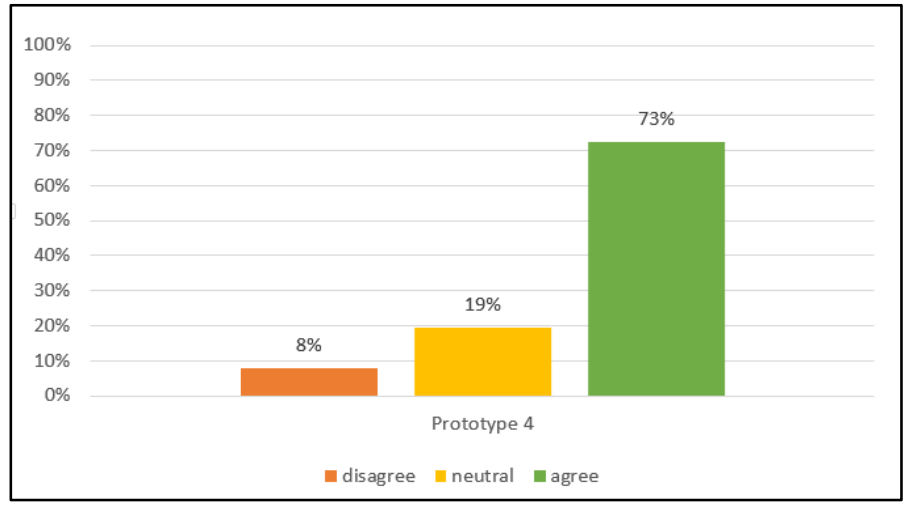

Graph 4. Were there advantages in using ARG in educational context?

\section{Conclusion and Future Work}

From the results of the questionnaires completed by students at the end of each cycle, the analysis of the opinions shows that the students were highly interested with the possibility of interacting with the programmatic contents, perceived and valued the use of devices with $\mathrm{AR}$ in the acquisition of the subject contents, and demonstrated that the use of the 
prototypes added an extra and relevant motivation to solve the tasks proposed by the teacher. During the use of the prototypes, the students still showed high levels of concentration when solving the tasks with the use of the prototypes. The high levels of concentration observed in students, when using prototypes, may be due to the introduction of new teaching methods, which they are not used to. Thus, diversificating the teaching methodologies can be an enriching experience and a relevant factor forthe success of learning. Although this type of technology is not yet sufficiently used in education, the enthusiasm of the students can be a determining factor for the success of the use of AR in the teaching-learning process.

The work continues with deeper analysis on the gathered data. A preliminary analysis of the results shows a promising field of research. The following stage in this research is to analyse the teachers opinions, after using the prototypes, to verify their perception on the added value that AR can have in the teaching and learning process. At the same time identify, by the opinions of teachers, new aproaches for teaching methodologies.

\section{Acknowledgements}

We are grateful to the research unit DIGIMEDIA at Aveiro University for its support in attending HEAd' 18 conference.

\section{References}

Brophy, J. E. (2004). Motivating students to learn - second edition. Routledge.

Di Serio, Á., Ibáñez, M. B., \& Kloos, C. D. (2013). Impact of an augmented reality system on students' motivation for a visual art course. Computers \& Education, 68, 586-596.

Joseph, J. (2012). The barriers of using education technology for optimizing the educational experience of learners. Procedia-Social and Behavioral Sciences, 64, 427-436.

Kesim, M., \& Ozarslan, Y. (2012). Augmented reality in education: current technologies and the potential for education. Procedia-Social and Behavioral Sciences, 47, 297-302.

Nincarean, D., Alia, M. B., Halim, N. D. A., \& Rahman, M. H. A. (2013). Mobile augmented reality: The potential for education. Procedia-Social and Behavioral Sciences, 103, 657-664. ${ }^{\circ}$

Shelton, B. E., \& Hedley, N. R. (2002). Using augmented reality for teaching earth-sun relationships to undergraduate geography students. Augmented Reality Toolkit, The First IEEE International Workshop (pp. 8-pp).

Sobral, S. R., \& Menezes, N. D. C. A. P. (2012). Motivação de alunos com e sem utilização das TIC em sala de aula. 